\title{
Deletion, Bell's Inequality, Teleportation
}

\author{
Indranil Chakrabarty ${ }^{1,3 *}$, \\ Nirman Ganguly ${ }^{1,2}$,Binayak S. Choudhury ${ }^{2}$ \\ ${ }^{1}$ Heritage Institute of Technology, Kolkata - 107, WestBengal, India. \\ ${ }^{2}$ Bengal Engineering and Science University, Shibpur, West Bengal, India. \\ ${ }^{3}$ Institute of Physics, Sachivalaya Marg, 751005 Bhubaneswar, Orissa, India
}

\begin{abstract}
In this letter we analyze the efficacy of the entangled output of Pati-Braunstein deletion machine [3] as a teleportation channel. We analyze the possibility of it violating the Bell's inequality. Interestingly we find that for all values of the input parameter $\alpha$ the state does not violate the Bell's inequality but when used as a teleportation channel can give a fidelity higher than the classical optimum (i.e $\frac{2}{3}$ ).
\end{abstract}

\section{Introduction:}

The complementary theory of 'quantum no-cloning theorem' [1] is the 'quantum nodeleting' principle [2]. It states that if we have two identical qubits at the input port, then there does not exist any linear map that will delete unknown quantum state against a copy. Quantum deletion [2] is more like reversible uncopying of an unknown quantum state. When memory in a quantum computer is scarce, quantum deleting may play an important role. The no-deleting principle does not prohibit us from constructing the approximate deleting machine $[3,4,5,6]$. In [3] authors constructed a input state state

*Corresponding author: E-Mail-indranilc@indiainfo.com 
dependent deletion machine. Later in [5] authors have constructed an universal deletion machine by making different fidelities free from the probability amplitude of the input state.

In his pioneering work, Bell [7] proved that, in general, two quantum states cannot be considered as separate even if they are located far from each other. When measurements are performed independently on each of the systems, their results are correlated in a way which cannot be explained by any local model. Although Bell's inequalities witness entanglement but there are entangled states which do not violate Bell's inequalities. Werner [8] gave an example of an entangled state described by the density operator $\rho_{W}=p\left|\psi^{-}\right\rangle\left\langle\psi^{-}\right|+\frac{1-p}{4} I$, where $\left|\psi^{-}\right\rangle=\frac{1}{\sqrt{2}}(|01\rangle-|10\rangle)$ and I is the identity operator in the 4-dimensional Hilbert space, which does not violate the Bell's inequality for $\frac{1}{3}<p<\frac{1}{\sqrt{2}}$. Interestingly in this work, we also find an example of an entangled state that does not violate the Bell's inequality.

A milestone application of quantum information theory "Quantum Teleportation" was proposed in [9]. The basic idea is to use a pair of particles in a singlet state shared by distant partners Alice and Bob to perform successful teleportation of an arbitrary qubit from the sender Alice to the receiver Bob. Popescu [10] noticed that the pairs in a mixed state could be still useful for teleportation.

A natural question arises in concern with teleportation whether states which violate BellCHSH inequalities are suitable for teleportation. Horodecki et al [11] showed that any mixed two spin- $\frac{1}{2}$ state which violates the Bell-CHSH inequalities is suitable for teleportation. It was shown that for any state which violates Bell inequalities, $M(\rho)>1$, where $M(\rho)=\max _{i>j}\left(u_{i}+u_{j}\right)$, where $u_{i}$ are eigenvalues of the matrix $T^{\dagger} T$ [11]. At this point Popescu raised an important question, 'What is the exact relationship between Bell's inequality violation and teleportation? Since the exact relationship between Bell's inequality and teleportation is unknown, it remains interesting to see whether there exists any entangled state which does not violate Bell's inequality but still can act as a teleportation channel with a fidelity $>\frac{2}{3}$ In our recent work we have showed that the output entangled state of Buzek-Hillery cloning machine [12] satisfy Bell's inequality and at the same time can be used as a teleportation channel for certain range of the machine 
parameter [13].

Here in this work the basic motivation is to find such an entangled state which inspite of being consistent with the local realist model proposed by John Bell can exhibit the extreme non local phenomenon like teleportation. In that context we study the output of Pati-Braunstein deletion machine [3] and show that this state can be a perfect example of an entangled state that can act as a teleportation channel without violating Bell's

inequality. In a nutshell here in this work we exemplify the existence of special class of entangled state which in spite of satisfying Bell's inequality can act as a teleportation channel. In other way round we analyze the output of Pati- Braunstein deletion machine and consequently establish its utility as a resource of quantum teleportation.

\section{Pati-Braunstein Deletion Machine and output:}

Here we give a brief introduction to Pati-Braunstein deletion machine. Later we analyze its output and subsequently show that this output density can be utilized as a resource for faithful teleportation.

For orthogonal qubits, the action of such a machine is given by,

$$
\begin{aligned}
& |0\rangle|0\rangle|A\rangle \longrightarrow|0\rangle|\Sigma\rangle\left|A_{0}\right\rangle \\
& |0\rangle|1\rangle|A\rangle \longrightarrow|0\rangle|1\rangle|A\rangle \\
& |1\rangle|0\rangle|A\rangle \longrightarrow|1\rangle|0\rangle|A\rangle \\
& |1\rangle|1\rangle|A\rangle \longrightarrow|1\rangle|\Sigma\rangle\left|A_{1}\right\rangle
\end{aligned}
$$

where $|A\rangle$ is the initial machine state and $\left|A_{0}\right\rangle,\left|A_{1}\right\rangle$ are the final states of ancilla. $|\Sigma\rangle=m_{1}|0\rangle+m_{2}|1\rangle,\left(m_{1}^{2}+m_{2}^{2}=1\right)$ is the blank state.

For an arbitrary pair of qubits the action of such a transformation is given by,

$$
\begin{array}{r}
|\Psi\rangle|\Psi\rangle|A\rangle=\left[\alpha^{2}|00\rangle+\beta^{2}|11\rangle+\alpha \beta(|0\rangle|1\rangle+|1\rangle|0\rangle)\right]|A\rangle \\
\left.\rightarrow \alpha^{2}|0\rangle|\Sigma\rangle\left|A_{0}\right\rangle+\beta^{2}|1\rangle|\Sigma\rangle\left|A_{1}\right\rangle+\alpha \beta(|0\rangle|1\rangle+|1\rangle|0\rangle)\right]|A\rangle \\
=\left|\Psi_{\text {out }}\right\rangle
\end{array}
$$


Here the ancilla states $|A\rangle,\left|A_{0}\right\rangle$ and $\left|A_{1}\right\rangle$ are orthogonal to each other. The reduced density matrix of the two qubits after the deletion operation is:

$$
\rho_{a b}=|\alpha|^{4}|0\rangle\langle 0|\otimes| \Sigma\rangle\left\langle\left.\Sigma|+| \beta\right|^{4} \mid 1\right\rangle\langle 1|\otimes| \Sigma\rangle\left\langle\left.\Sigma|+2| \alpha\right|^{2}|\beta|^{2} \mid \psi^{+}\right\rangle\left\langle\psi^{+}\right|
$$

We now analyze the output (3) to investigate its inseparable nature for different values of input parameters $\alpha$ and $\beta$ and subsequently look for its efficiency as a teleportation channel. For simplicity here we assume the initial state $|\psi\rangle$ and blank state $|\Sigma\rangle$ to be a member of real Hilbert Space, by taking $\alpha, \beta, m_{1}$ and $m_{2}$ as real quantities.

\section{i) Inseparability of the output:}

The necessary and sufficient condition for the state $\rho$ of two spin $\frac{1}{2}$ to be inseparable is that at least one of the eigenvalues of the partially transposed operator defined as $\rho_{m \mu, n \nu}^{T_{B}}=\rho_{m \nu, n \mu}$, is negative $[14,15]$. This is equivalent to the condition that at least one of the two determinants.

$W_{3}=\left|\begin{array}{lll}\rho_{00,00} & \rho_{01,00} & \rho_{00,10} \\ \rho_{00,01} & \rho_{01,01} & \rho_{00,11} \\ \rho_{10,00} & \rho_{11,00} & \rho_{10,10}\end{array}\right|$ and $W_{4}=\left|\begin{array}{cccc}\rho_{00,00} & \rho_{01,00} & \rho_{00,10} & \rho_{01,10} \\ \rho_{00,01} & \rho_{01,01} & \rho_{00,11} & \rho_{01,11} \\ \rho_{10,00} & \rho_{11,00} & \rho_{10,10} & \rho_{11,10} \\ \rho_{10,01} & \rho_{11,01} & \rho_{10,11} & \rho_{11,11}\end{array}\right|$

is negative.

After calculating the determinants for $\rho_{a b}$, we obtain the values of $W_{3}$ and $W_{4}$ as

$$
W_{3}=\alpha^{6} \beta^{4} m_{1}^{2}\left(\alpha^{2}+m_{1}^{2} \beta^{2}\right), \quad W_{4}=-\alpha^{6} \beta^{6}\left[\alpha^{4} m_{2}^{2}+m_{1}^{2} \beta^{4}+\alpha^{2} \beta^{2}\right]
$$

It is evident that $W_{3}>0 \quad\left(\forall \alpha, \beta, m_{1}, m_{2}\right)$ and $W_{4}<0 \quad\left(\forall \alpha, \beta, m_{1}, m_{2}\right)$. Since at least one of these two determinants is less than zero, hence we with full generality conclude the state $\rho_{a b}$ to be inseparable.

\section{ii) Non-Violation of Bell's Inequality of two qubit entangled state:}

For simplicity we assume the blank state as $|\Sigma\rangle=\frac{1}{\sqrt{2}}(|0\rangle+|1\rangle)$, by taking $m_{1}=m_{2}=\frac{1}{\sqrt{2}}$. It is a known fact that the state which does not violate Bell's inequality must satisfy $M(\rho) \leq 1$, where $M(\rho)=\max _{i>j}\left(u_{i}+u_{j}\right)$ where $u_{i}$ and $u_{j}$ are the eigenvalues of $U=$ $C^{t}(\rho) C(\rho)$ where $C(\rho)=\left[C_{i j}\right], C_{i j}=\operatorname{Tr}\left[\rho \sigma_{i} \otimes \sigma_{j}\right][11]$.

The eigenvalues of the matrix $U=C^{t}\left(\rho_{a b}\right) C\left(\rho_{a b}\right)$ for the bipartite output state $\rho_{a b}$ of P-B deleting machine are given by,

$$
u_{1}=4 \alpha^{4}-8 \alpha^{6}+4 \alpha^{8}
$$




$$
\begin{aligned}
& u_{2}=A+\frac{1}{2} \sqrt{B} \\
& u_{3}=A-\frac{1}{2} \sqrt{B} \\
& \text { where } A=4 \alpha^{8}-8 \alpha^{6}+6 \alpha^{4}-2 \alpha^{2}+\frac{1}{2}, \\
& B=1+64 \alpha^{12}+224 \alpha^{8}-8 \alpha^{2}-192 \alpha^{10}-128 \alpha^{6}+40 \alpha^{4}
\end{aligned}
$$

A simple calculation will reveal that out of these three eigenvalues, $u_{1}, u_{2}$ are the largest two. So taking these two eigenvalues into consideration the expression for $M(\rho)$ will be less than 1 for all values of $\alpha$ lying in the range $(0,1)$.

\section{iii) Efficiency of the two qubit entangled state as teleportation channel:}

Next we investigate whether the entangled state $\rho_{a b}$ can act as a teleportation channel.

Let us recall the eigenvalues of the matrix $U=C^{t}\left(\rho_{a b}\right) C\left(\rho_{a b}\right), u_{1}, u_{2}$ and $u_{3}$ given in (5). Hence the teleportation fidelity $F_{\max }[11]$ is given by,

$$
F_{\max }=\frac{1}{2}\left[1+\frac{\sqrt{u_{1}}+\sqrt{u_{2}}+\sqrt{u_{3}}}{3}\right]
$$

Next we find out the values of $F_{\max }$ for different values of the input parameter $\alpha$ and enlist down in the following table.

\section{TABLE 1:}

\begin{tabular}{|c|c|}
\hline$\alpha$ & $F_{\max }$ \\
\hline 0.1 & 0.666783 \\
\hline 0.2 & 0.668531 \\
\hline 0.3 & 0.675915 \\
\hline 0.4 & 0.694094 \\
\hline 0.5 & 0.725347 \\
\hline 0.6 & 0.765805 \\
\hline 0.7 & 0.808094 \\
\hline 0.8 & 0.847683 \\
\hline 0.9 & 0.893974 \\
\hline
\end{tabular}

Interestingly we find $F_{\max } \geq \frac{2}{3} \forall \alpha \in(0,1)$.

A particular case : $\alpha=\beta=\frac{1}{\sqrt{2}}$ 
Here we consider the case when the input state is an equal superposition of qubits. For $\alpha=\beta=\frac{1}{\sqrt{2}}$, the eigenvalues of $\mathrm{U}$ matrix are given by,

$$
u_{1}=u_{2}=u_{3}=\frac{1}{4}
$$

The teleportation fidelity is

$$
F_{\max }=\frac{3}{4}
$$

Hence the output can be used as a teleportation channel $\forall \alpha \in(0,1)$. Thus we see that the output of P-B deleting machine can be used as a resource to quantum teleportation. Not only that we see this output state gives a positive response to our search for an entangled state that does not violate Bell's inequality and still can act as a teleportation channel.

\section{Conclusion :}

In foundation of quantum mechanics violation of Bell's inequality is considered to be a signature of the existence of non local properties of the entangled states. At the same time quantum teleportation (entangled states acting as teleportation channel with a fidelity $>\frac{2}{3}$ ) itself is a manifestation of non locality. Combining these two we can say that there is no such elements of surprise in finding out entangled states that are violating Bell's inequality and still can act as a teleportation channel. But it remains interesting when we find out an entangled state that does not violate Bell's inequality and still can act as a teleportation channel. The basic motivation of the work is to find out such an entangled state. Here we have cited an example of a two qubit entangled state (output of the Pati-Braunstein deleting machine) [3] which does not violate Bell-CHSH inequality [9] and at the same time can act as a useful quantum channel for teleportation protocol. This work adds a special feature to the Pati- Braunstein quantum deleting machine by demonstrating its output state as a potential resource for quantum teleportation. 


\section{Acknowledgement:}

I.C acknowledges Prof C.G.Chakraborti for being the source of inspiration in research work. N.G acknowledges his mother for her love and blessings.

\section{Reference:}

[1] W.K.Wootters and W.H.Zurek,Nature 299,802(1982).

[2] A.K.Pati and S.L.Braunstein Nature 404, 164 (2000) .

[3] A.K.Pati and S.L.Braunstein, arxiv:quant-ph/0007121v1(2000)

[4] D.Qiu, Phys.Lett.A 301,112 (2002).

[5] S.Adhikari, Phys. Rev. A 72, 052321 (2005).

[6] S. Adhikari and B. S. Choudhury, Phys. Rev. A 73, 054303 (2006).

[7] J.S.Bell , Physics 1 (1964) 195

[8] R.F.Werner, Phys.Rev.A 40, 4277 (1989).

[9] C.Benett,G.Brassard,C.Crepeau,R.Jozsa,A.peres and W.K.Wootters, Phys Rev Lett 70(1993) 1895.

[10] S.Popescu, Phys.Rev.Lett. 72, 797 (1994).

[11] R.Horodecki, M.Horodecki, P.Horodecki, Phys.Lett.A 222, 21 (1996).

[12] V. Buzek, M. Hillery, Phys. Rev.A 54, 1844 (1996).

[13] S. Adhikari, N. Ganguly, I. Chakrabarty, B. S. Choudhury, J. Phys. A: Math. Theor. 41415302 (2008).

[14] A.Peres, Phys.Rev.Lett. 77, 1413 (1996).

[15] M.Horodecki, P.Horodecki, R.Horodecki, Phys.Lett.A 223, 1 (1996). 